\title{
Association of Plasma IL-32 Levels and Gene Polymorphisms with Systemic Lupus Erythematosus in Chinese Han Population
}

\author{
Yanyun Wang, ${ }^{1}$ Bin Zhou, ${ }^{1}$ Yi Zhao, ${ }^{2}$ Xiuzhang Yu, ${ }^{3}$ Yi Liu, ${ }^{2}$ and Lin Zhang ${ }^{1}$ \\ ${ }^{1}$ Laboratory of Molecular and Translational Medicine, Key Laboratory of Birth Defects and Related Diseases of Women and \\ Children of Ministry of Education at Sichuan University, West China Second University Hospital, Sichuan University, \\ Chengdu, Sichuan 610061, China \\ ${ }^{2}$ Department of Rheumatology and Immunology, West China Hospital, Sichuan University, Chengdu, Sichuan 610041, China \\ ${ }^{3}$ Department of Obstetrics and Gynecology, West China Second University Hospital, Sichuan University, \\ Chengdu, Sichuan 610061, China
}

Correspondence should be addressed to Yi Liu; yi2006liu@163.com and Lin Zhang; zhanglin@scu.edu.cn

Received 2 December 2015; Revised 26 January 2016; Accepted 9 February 2016

Academic Editor: Fabrizia Bamonti

Copyright (C) 2016 Yanyun Wang et al. This is an open access article distributed under the Creative Commons Attribution License, which permits unrestricted use, distribution, and reproduction in any medium, provided the original work is properly cited.

\begin{abstract}
Systemic lupus erythematosus (SLE) is a multisystemic autoimmune disease. IL-32, a secreted protein, has been reported to be associated with several autoimmune diseases. Our preliminary experiment showed different plasma IL-32 levels than that mentioned in a published report on the same population. In order to elucidate the correlation between IL-32 and SLE, we determined the plasma level and two single nucleotide polymorphisms (SNPs) of IL-32 in 152 patients with SLE and 310 healthy controls and analyzed the relationship based on the clinical parameters. The results showed that plasma IL-32 levels in patients with SLE were markedly lower than that in the healthy controls. In the SLE group, patients with detectable IL-32 presented low serum C3 concentrations. Further studies indicated that the rs 28372698 SNP was associated with the susceptibility to SLE. Taken together, our results suggested that IL-32 could possibly be a candidate marker to monitor SLE disease stability and screening in future.
\end{abstract}

\section{Introduction}

Systemic lupus erythematosus (SLE) is a multisystemic autoimmune disease, which involves multiple organ and tissue injuries [1]. The prevalence rate of SLE is about 70 cases per 100,000 people in the Chinese population [2]. Cytokines such as TNF $\alpha$, interleukin 6 (IL-6), IL-27, and IL-12 [3-5] have been reported to be associated with SLE pathogenesis.

IL-32 is a multifunctional secreted protein that plays important roles in antimicrobial pathways $[6,7]$, cancer $[8,9]$, and autoimmune diseases, including rheumatoid arthritis (RA), myasthenia gravis, and giant cell arteritis [10-13].

To date, only few studies have focused on the association of SLE and IL-32 [14, 15]. Zhang et al. showed that serum IL32 level was not statistically different between patients with SLE and healthy controls in the Chinese population [14]. In a preliminary experiment, our team showed that the plasma IL32 level in healthy controls was markedly different than that observed by Zhang et al. (39.25 (21.00-70.46) pg/mL). Such a difference within the same population seemed abnormal. In the present study, we aimed to better understand the correlation between SLE and IL-32 using serology and immunogenetics in a larger sample size of the Chinese population.

\section{Materials and Methods}

2.1. Subjects. One hundred and fifty-two female patients with SLE (16-60 years old) and 310 healthy female controls (18-59 years old) were enrolled in this study. Patients with SLE were recruited from West China Hospital of Sichuan University. The diagnosis of SLE was confirmed according to the 1997updated SLE criteria wrote by the American College of Rheumatology [16]. SLE disease activity index (SLEDAI) was scored by two independent doctors from the Department of Rheumatology at West China Hospital of Sichuan University. Healthy controls were from a routine health checkup in West China Second University Hospital of Sichuan University. 
TABLE 1: Characteristics of the patients.

\begin{tabular}{lc}
\hline & Patients \\
\hline Mean duration of disease (months) & $1-156$ \\
SLEDAI $(n)$ & \\
$0-9$ score & 94 \\
$\geqslant 10$ score & 58 \\
Lupus nephritis $(n)$ & \\
$\quad$ Yes & 105 \\
No & 47 \\
24 h urinary protein $(n)$ & \\
$\quad<0.5 \mathrm{~g}$ & 94 \\
$\geqslant 0.5 \mathrm{~g}$ & 58 \\
C3 $(n)$ & \\
$\quad<0.785 \mathrm{~g} / \mathrm{L}$ & 96 \\
$\geqslant 0.785 \mathrm{~g} / \mathrm{L}$ & 56 \\
C4 $(n)$ & \\
$\quad<0.145 \mathrm{~g} / \mathrm{L}$ & 69 \\
$\geqslant 0.145 \mathrm{~g} / \mathrm{L}$ & 83 \\
Anti-dsDNA $(n)$ & \\
Positive & 65 \\
Negative & 87 \\
Anti-ANA $(n)$ & \\
Positive & \\
Negative & \\
\hline
\end{tabular}

They had no personal or family history of cancer, autoimmune diseases, or other serious diseases. The characteristics of the SLE group were shown in Table 1. This study was approved by the Ethics Committees of West China Hospital of Sichuan University and West China Second University Hospital of Sichuan University. All subjects wrote the informed consent.

2.2. Sample Collection. Peripheral blood of all subjects was collected into EDTA-containing vacuum blood collection tube. Samples were centrifuged at $1600 \mathrm{rpm}$ for 10 minutes at $4^{\circ} \mathrm{C}$. Plasma was aliquoted and stored at $-80^{\circ} \mathrm{C}$. Genomic DNA was extracted by a DNA isolation kit from Bioteke (Peking, Beijing, China).

2.3. Detection of Plasma IL-32 Level Using an EnzymeLinked Immunosorbent Assay (ELISA). Plasma IL-32 levels were measured using a commercially available sandwich ELISA kit (DY3040-05, R\&D Systems, Minneapolis, MN, USA) according to the manufacturer's protocol. According to the product manual, the kit recognizes human IL-32 $\alpha$, IL-32 $\beta$, and IL-32 $\gamma$. The whole procedure was performed at room temperature (RT). In brief, 96-well microplates were coated with $100 \mu \mathrm{L}$ per well of the diluted human IL-32 capture antibody. After incubation overnight, plates were washed and then blocked by Reagent Diluent (DY995, R\&D Systems, Minneapolis, USA) for 1 hour. The plates were ready for use after washed. Each plasma sample was added to duplicate wells (100 $\mu \mathrm{L}$ per well) and incubated 2 hours. The plates were washed. $100 \mu \mathrm{L}$ of diluted human IL-32 detection antibody was then added to each well for incubating 2 hours. The plates were washed again. Streptavidin-HRP was added for incubating 20 minutes. Color development was carried out by addition of $100 \mu \mathrm{L}$ substrate solution (DY999, R\&D Systems, Minneapolis, USA). The optical density of each well was determined using a multimode microplate reader (M200, TECAN Infinite, Switzerland) which was set to $450 \mathrm{~nm}$ as measurement wavelength and $540 \mathrm{~nm}$ as reference wavelength. The four-parameter logistic standard curve was generated using SigmaPlot software version 12.0 (Systat Software Inc., California, USA). The plasma IL-32 concentration was determined by standard curve.

2.4. Genotyping of Two Genetic Variants of $I L-32$. In the present study, two single nucleotide polymorphisms (SNPs), rs12934561 and rs28372698, were genotyped. The experiments were performed as described previously [17]. About $10 \%$ of the samples were randomly selected to perform the DNA sequencing analysis. The results were $100 \%$ concordant.

2.5. Statistical Analyses. The test of normal distribution of plasma IL-32 levels was conducted by SPSS software version 13.0 (SPSS Inc., Chicago, IL, USA). The plasma IL-32 level was described in the form of median (interquartile range). A nonparametric test (Mann-Whiney test) was used to compare plasma IL-32 levels between patients with SLE and healthy controls. Genotype frequencies were determined by directed counting. Hardy-Weinberg equilibrium, genotype, and allele association were performed by chi-square test. Odds ratio (OR) and respective 95\% confidence intervals (95\% CIs) were used to evaluate the effects of any difference between genotype and alleles. $P<0.05$ (two-sided test) was considered to be statistically significant.

\section{Results}

3.1. Comparison of Plasma IL-32 Levels between Patients with SLE and Healthy Controls. Plasma IL-32 levels of 104 patients with SLE and 107 healthy controls were measured by ELISA. Plasma IL-32 levels were detectable in 80 healthy controls and 21 patients with SLE. IL-32 concentration in the plasma was $34.72(15.45-140.54) \mathrm{pg} / \mathrm{mL}$ in patients with SLE and 94.40 (40.21-233.73) pg/mL in the healthy controls (Figure 1). The difference was statistically significant $(P=0.02)$.

Several clinical characteristics were compared between the subgroup of SLE patients with detectable IL-32 and the subgroup in which IL-32 was nondetectable. Serum C3 levels were significantly different between the patients with detectable IL-32 and patients with nondetectable IL$32(P=0.0215)$. However, no correlation with SLEDAI score, proteinuria, serum $\mathrm{C} 4$, anti-dsDNA, and anti-ANA was observed (Table 2). Among the patients with detectable IL32, the plasma level of IL-32 was not correlated with SLEDAI score, proteinuria, serum $\mathrm{C} 3$, serum $\mathrm{C} 4$, anti-dsDNA, and anti-ANA.

Then the relationship between treatment methods and IL32 level was analyzed. The results explored that IL-32 level was not associated with the treatment methods which the patients were suffering from when the samples were collected (small 
TABLE 2: Clinical Characteristics and genotype of two SNPs of patients whose plasma levels of IL-32 were measured.

\begin{tabular}{|c|c|c|c|}
\hline & IL-32 detectable $(n=21)$ & IL-32 not detectable $(n=83)$ & $P$ value \\
\hline Age (mean $\pm S D)$, years & $37 \pm 10.58$ & $35.17 \pm 11.12$ & \\
\hline Mean duration of disease (months) & $6-144$ & $1-156$ & \\
\hline \multicolumn{4}{|l|}{$\operatorname{SLEDAI}(n)$} \\
\hline $0-9$ score & 16 & 62 & \multirow{2}{*}{1.000} \\
\hline$\geqslant 10$ score & 5 & 21 & \\
\hline \multicolumn{4}{|l|}{ Lupus nephritis ( $n$ ) } \\
\hline Yes & 16 & 59 & \multirow{2}{*}{0.7880} \\
\hline No & 5 & 24 & \\
\hline \multicolumn{4}{|l|}{24 h urinary protein $(n)$} \\
\hline$<0.5 \mathrm{~g}$ & 13 & 59 & \multirow{2}{*}{0.4360} \\
\hline$\geqslant 0.5 \mathrm{~g}$ & 8 & 24 & \\
\hline \multicolumn{4}{|l|}{$\mathrm{C} 3(n)$} \\
\hline$<0.785 \mathrm{~g} / \mathrm{L}$ & 20 & 58 & \multirow{2}{*}{$0.0215^{*}$} \\
\hline$\geqslant 0.785 \mathrm{~g} / \mathrm{L}$ & 1 & 25 & \\
\hline \multicolumn{4}{|l|}{$\mathrm{C} 4(n)$} \\
\hline$<0.145 \mathrm{~g} / \mathrm{L}$ & 15 & 51 & \multirow{2}{*}{0.4560} \\
\hline$\geqslant 0.145 \mathrm{~g} / \mathrm{L}$ & 6 & 32 & \\
\hline \multicolumn{4}{|l|}{ rs28372698 genotype $(n)$} \\
\hline AA & 7 & 39 & \multirow{3}{*}{0.3045} \\
\hline AT & 12 & 32 & \\
\hline TT & 2 & 12 & \\
\hline \multicolumn{4}{|l|}{ rs12934561 genotype $(n)$} \\
\hline TT & 13 & 30 & \multirow{3}{*}{0.1010} \\
\hline TC & 5 & 33 & \\
\hline $\mathrm{CC}$ & 3 & 20 & \\
\hline
\end{tabular}

${ }^{*}$ Statistically significant.

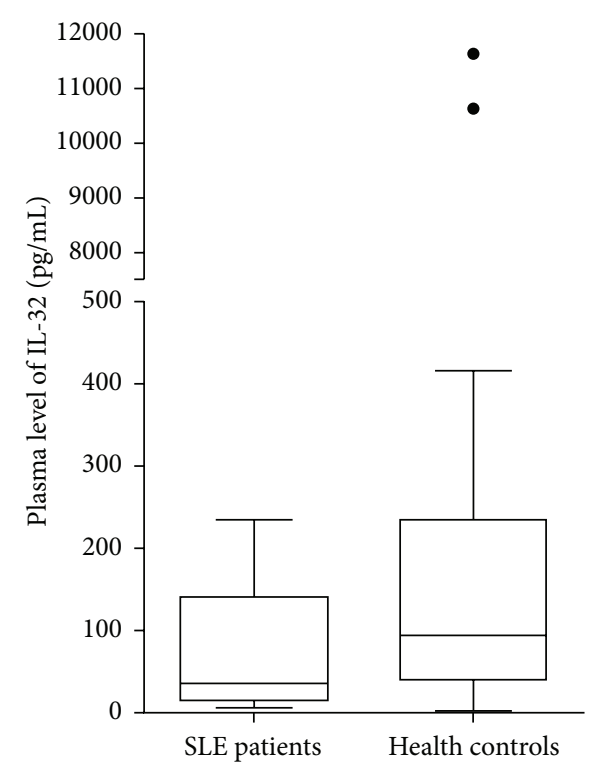

FIgURE 1: The plasma level of IL-32 in SLE patients and health controls.

dose prednisolone plus hydroxychloroquine or prednisolone plus cyclophosphamide or mycophenolate) (data not shown).

3.2. Detection of IL-32 SNPS and Susceptibility to SLE. rs12934561 and rs28372698 SNPs were successfully genotyped in all subjects. The genotype frequencies of both SNPs were in agreement with the Hardy-Weinberg equilibrium $(P<$ $0.05)$. The results are presented in Table 3 . With regard to rs 28372698 , the TT genotype was associated with increased risk of SLE $(P=0.011, \mathrm{OR}=2.32$, and $95 \% \mathrm{CI}=1.20-4.50$ 


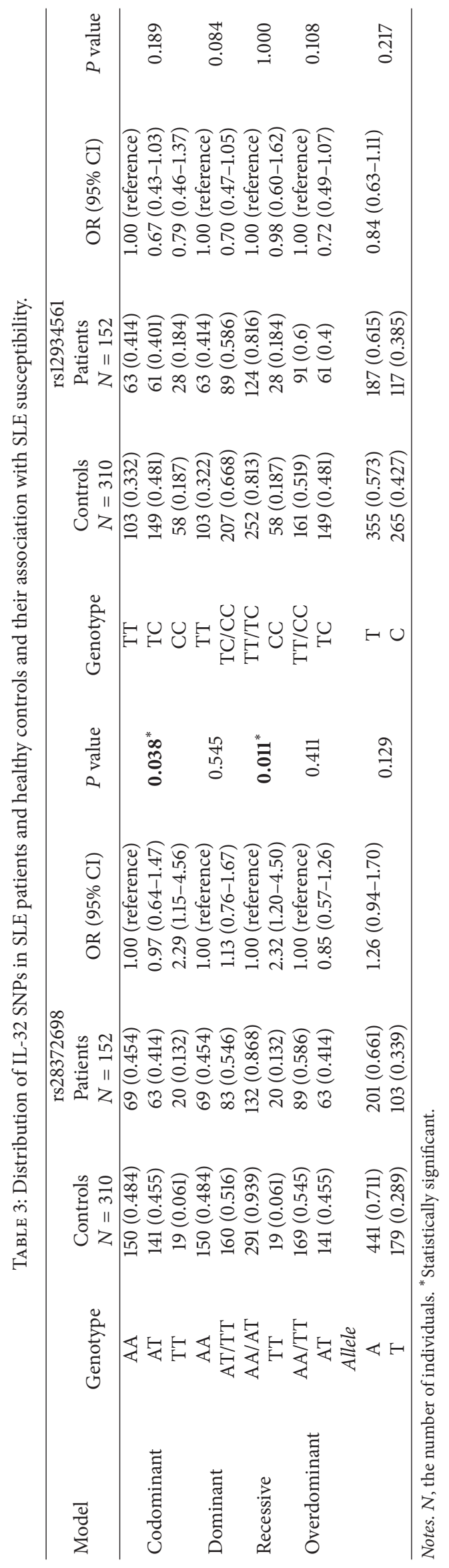


in the recessive model), while the allele frequency was not statistically different between the patients and the healthy controls. Allele and genotype distribution frequencies of rs12934561 showed no significant difference between patients with SLE and healthy controls.

3.3. Relationship between Plasma IL-32 Levels and IL-32 SNPS in Patients with SLE. The relationship between plasma IL32 levels and IL-32 SNPs was analyzed in order to explore if genetic variants could affect plasma IL-32 levels. There was no correlation between the genotype and the plasma IL-32 levels (Table 2).

\section{Discussion}

IL-32 is considered as a proinflammatory cytokine, which is related to IL-1 $\beta$, IL-18, IL-21, and IL-23. It has been reported to play important roles in the pathogenesis of various autoimmune diseases. Gui et al. showed that the level of IL-32 in the plasma was significantly associated with RA disease activity [10]. Another study suggested that IL-32 might be correlated with the pathogenesis or immunoregulation of myasthenia gravis [11].

Only two studies on the association between SLE and IL32 were found in the PubMed database (http://www.ncbi.nlm .nih.gov/pubmed/). Zhang et al. showed that serum IL-32 concentrations in patients with SLE were not different from that in the healthy controls in a Chinese population [14]. Although both our study and that of Zhang et al. focused on the Chinese population, our study was markedly different from that of Zhang et al. In the present study, plasma IL32 levels in patients with SLE were lower than those of the controls. There are six IL-32 isoforms (IL-32 $\alpha$, IL-32 $\beta$, IL-32 $\gamma$,

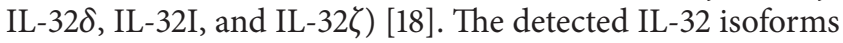
were not described clearly in the study by Zhang et al. Thus, we cannot be sure that the difference observed in the same population was caused by different indices of detection.

We speculate that the lower plasma IL-32 level in the SLE group may be associated with drug treatment. In fact, the concentration of serum IL-17 could be reduced after cyclophosphamide treatment for 4 weeks in patients with SLE and IL-17 is known to affect the expression of IL-32 [19, 20]. Majority of the patients with SLE (enrolled in our study) were taking medicine for at least six months. Therefore, IL32 levels may have changed. Additionally, IL-32 was shown to be associated with the chemotherapy-related bone marrow cytotoxicity [21]. The arrest of bone marrow was the most common immunosuppressant side-effect for patients with SLE. This might be another possible reason for decreased IL32 levels. Although the treatment methods did not associate with the IL-32 detection and level in our present study, in the future, the SLE patients without treatment would be needed to be enrolled in the follow-up study. It could be helpful in better exploring the correlation between treatment and IL-32.

In the present study, plasma IL-32 was detectable in only $20.2 \%$ of the patients. Inoue et al. detected the serum level of IL-32 $\gamma$ in 51 patients with SLE and 15 healthy controls. The results demonstrated that IL-32 was detectable in only 3 patients and led to the speculation that IL-32 $\gamma$ possibly contributes to the pathogenesis of renal diseases in patients with SLE [15]. In our study, no correlation between plasma IL32 level and urinary proteins or complicated lupus nephritis was observed. However, serum C3 levels tended to be low in patients with detectable IL-32, suggesting that IL-32 could possibly be a candidate marker to monitor SLE disease stability and screening in future. As the more sensitive detection methods were improved, the nondetectable samples in the present study might be detected; the difference and association among SLE group might be explored more clearly.

Genetic factors, such as SNPs, have been reported to be associated with the corresponding serum level [22-24]. In order to explore if the different plasma IL-32 levels in patients with SLE were correlated with the genetic effect, two SNPs (rs12934561 and rs28372698) were detected in this study. rs12934561 is a C/T single nucleotide variation in an intron, which is associated with acute lung injury and endometrial cancer [17, 25]. rs 28372698 , a promoter SNP, is associated with the risk of gastric cancer and endometrial cancer $[17,26]$. We analyzed the correlation between the genotype of the patients for these two SNPs and plasma IL-32 levels. The result showed no significant association between plasma IL32 levels and the genotype.

Interestingly, the TT genotype of rs 28372698 was found to be associated with increased risk of SLE in the recessive model. However, the role of this polymorphism with regard to the function of IL-32 remains unknown due to the lack of corresponding reports. Future studies are warranted to investigate this question.

In summary, the present study explored the correlation between IL-32 polymorphism and SLE for the first time and provided new data regarding IL-32 levels in the plasma of a Chinese population. Taken together, our results suggested that IL-32 could possibly be a candidate marker to monitor SLE disease stability and screening in future.

\section{Conflict of Interests}

The authors have no conflict of interests to declare.

\section{Acknowledgments}

This work was supported by grants from National Natural Science Foundation of China (no. 81501261, no. 81272821, and no. 81172440). Thanks are due to Ms. Jiru Du and Ms. Yuan $\mathrm{Xu}$ for sample collection.

\section{References}

[1] R. A. Montes, L. O. Mocarzel, P. G. Lanzieri, L. M. Lopes, A. Carvalho, and J. R. Almeida, "Smoking and its association with morbidity in systemic lupus erythematosus evaluated by the Systemic Lupus International Collaborating Clinics/American College of Rheumatology damage index: preliminary data and systematic review," Arthritis \& Rheumatology, vol. 68, no. 2, pp. 441-448, 2016.

[2] Y. Rengao, Systemic Lupus Erythematosus. Internal Medicine, People's Medical Publishing House, Beijing, China, 2004. 
[3] A. Sabry, H. sheashaa, A. El-Husseini et al., "Proinflammatory cytokines (TNF- $\alpha$ and IL-6) in Egyptian patients with SLE: its correlation with disease activity," Cytokine, vol. 35, no. 3-4, pp. 148-153, 2006.

[4] A. L. B. P. Duarte, A. T. Dantas, H. de Ataíde Mariz et al., "Decreased serum interleukin 27 in Brazilian systemic lupus erythematosus patients," Molecular Biology Reports, vol. 40, no. 8, pp. 4889-4892, 2013.

[5] F. Qiu, L. Song, N. Yang, and X. Li, "Glucocorticoid downregulates expression of IL-12 family cytokines in systemic lupus erythematosus patients," Lupus, vol. 22, no. 10, pp. 1011-1016, 2013.

[6] D. Li, D. Chen, X. Zhang et al., "c-Jun N-terminal kinase and Akt signalling pathways regulating tumour necrosis factor- $\alpha$ induced interleukin-32 expression in human lung fibroblasts: implications in airway inflammation," Immunology, vol. 144, no. 2, pp. 282-290, 2015.

[7] D. Montoya, M. S. Inkeles, P. T. Liu et al., "IL-32 is a molecular marker of a host defense network in human tuberculosis," Science Translational Medicine, vol. 6, no. 250, Article ID 250ra114, 2014.

[8] A. Player, T. Oguamanam, J. Okanmelu, K. Burrell, and M. Hollomon, "Preliminary characterization of IL32 in basallike/triple negative compared to other types of breast cell lines and tissues," BMC Research Notes, vol. 7, no. 1, article 501, 2014.

[9] C. A. Nold-Petry, I. Rudloff, Y. Baumer et al., "IL-32 promotes angiogenesis," Journal of Immunology, vol. 192, no. 2, pp. 589$602,2014$.

[10] M. Gui, H. Zhang, K. Zhong, Y. Li, J. Sun, and L. Wang, "Clinical significance of interleukin-32 expression in patients with rheumatoid arthritis," Asian Pacific Journal of Allergy and Immunology, vol. 31, no. 1, pp. 73-78, 2013.

[11] S.-J. Na, S.-H. So, K. O. Lee, and Y.-C. Choi, "Elevated serum level of interleukin-32 $\alpha$ in the patients with myasthenia gravis," Journal of Neurology, vol. 258, no. 10, pp. 1865-1870, 2011.

[12] W.-D. Xu, M. Zhang, C.-C. Feng, X.-K. Yang, H.-F. Pan, and D.Q. Ye, "IL-32 with potential insights into rheumatoid arthritis," Clinical Immunology, vol. 147, no. 2, pp. 89-94, 2013.

[13] F. Ciccia, R. Alessandro, A. Rizzo et al., "Expression of interleukin-32 in the inflamed arteries of patients with giant cell arteritis," Arthritis and Rheumatism, vol. 63, no. 7, pp. 20972104, 2011.

[14] M. Zhang, W.-D. Xu, Y. Zhu et al., "Serum levels of cytokines in systemic lupus erythematosus: association study in a Chinese population," Zeitschrift für Rheumatologie, vol. 73, no. 3, pp. 277-280, 2014.

[15] M. Inoue, H. Shoda, Y. Seri et al., "Three cases of lupus nephritis patients with serum interleukin-32 $\gamma$ detection," Lupus, vol. 23, no. 11, pp. 1187-1191, 2014.

[16] M. C. Hochberg, "Updating the American College of Rheumatology revised criteria for the classification of systemic lupus erythematosus," Arthritis and rheumatism, vol. 40, article 1725, 1997.

[17] X. Yu, B. Zhou, Z. Zhang et al., "Significant association between IL-32 gene polymorphisms and susceptibility to endometrial cancer in Chinese Han women," Tumor Biology, vol. 36, pp. 5265-5272, 2015.

[18] J.-D. Choi, S.-Y. Bae, J.-W. Hong et al., "Identification of the most active interleukin-32 isoform," Immunology, vol. 126, no. 4, pp. 535-542, 2009.
[19] Y.-M. Moon, B.-Y. Yoon, Y.-M. Her et al., "IL-32 and IL-17 interact and have the potential to aggravate osteoclastogenesis in rheumatoid arthritis," Arthritis Research \& Therapy, vol. 14, no. 6, article R246, 2012.

[20] L. Zhao, H. Ma, Z. Jiang, Y. Jiang, and N. Ma, "Immunoregulation therapy changes the frequency of interleukin (IL)$22^{+} \mathrm{CD}^{+} \mathrm{T}$ cells in systemic lupus erythematosus patients," Clinical and Experimental Immunology, vol. 177, no. 1, pp. 212218, 2014.

[21] A. Moldenhauer, M. Futschik, H. Lu et al., "Interleukin 32 promotes hematopoietic progenitor expansion and attenuates bone marrow cytotoxicity," European Journal of Immunology, vol. 41, no. 6, pp. 1774-1786, 2011.

[22] B. Zietz, E. Watzlawek, K.-D. Palitzsch, J. Schölmerich, and A. Schäffler, "GG-genotype in the promotor region of uncoupling-protein-1 gene is associated with lower level of dehydroepiandrosterone in type 2 diabetes," Experimental and Clinical Endocrinology \& Diabetes, vol. 109, no. 2, pp. 102-106, 2001.

[23] M. I. Taskin, B. Eser, E. Adali, H. Kara, C. Cuce, and A. A. Hismiogullar1, "NUCB2 gene polymorphism and its relationship with nesfatin-1 levels in polycystic ovary syndrome," Gynecological Endocrinology, vol. 32, no. 1, pp. 46-50, 2015.

[24] N. M. Al-Daghri, O. S. Al-Attas, S. Krishnaswamy et al., "Association between promoter region genetic variants of PTH SNPs and serum 25(OH)-vitamin D level," International Journal of Clinical and Experimental Pathology, vol. 8, no. 7, pp. 84638471, 2015.

[25] J. J. Arcaroli, N. Liu, N. Yi, and E. Abraham, "Association between IL-32 genotypes and outcome in infection-associated acute lung injury," Critical Care, vol. 15, article R138, 2011.

[26] P. Gonzalez-Hormazabal, M. Musleh, M. Bustamante et al., "Role of cytokine gene polymorphisms in gastric cancer risk in Chile," Anticancer Research, vol. 34, no. 7, pp. 3523-3530, 2014. 


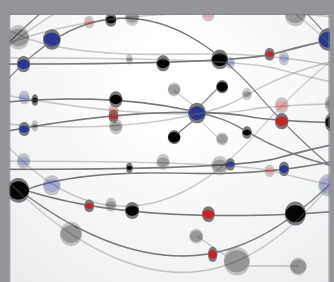

The Scientific World Journal
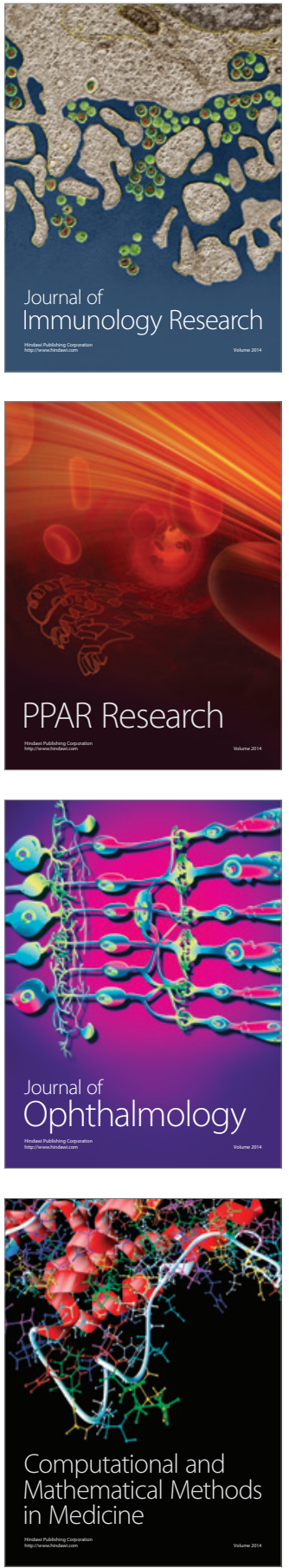

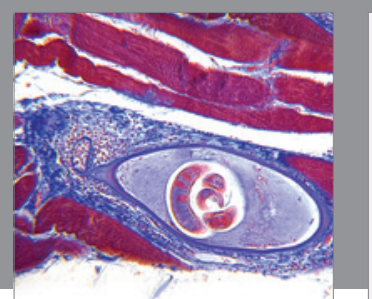

Gastroenterology Research and Practice

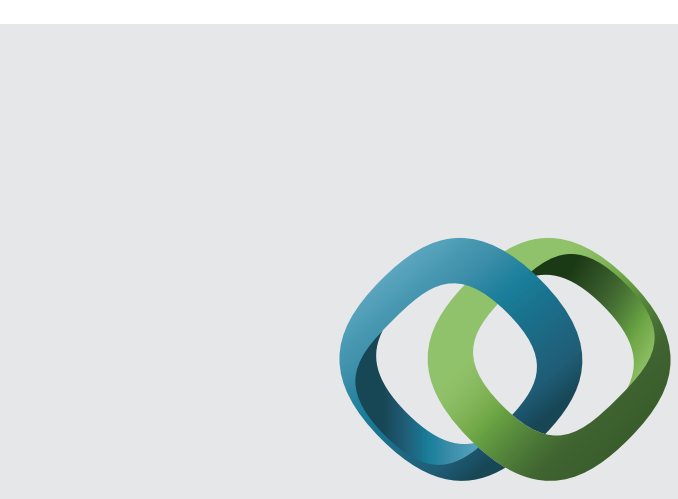

\section{Hindawi}

Submit your manuscripts at

http://www.hindawi.com
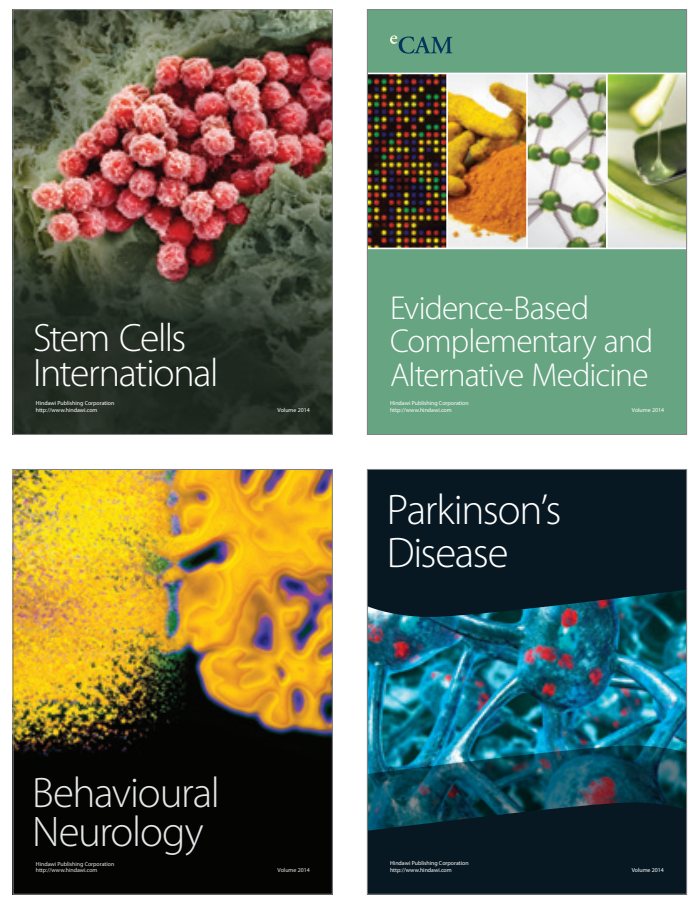
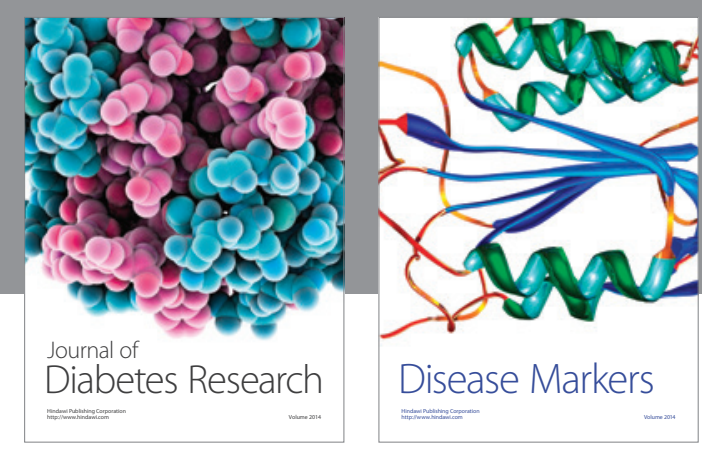

Disease Markers
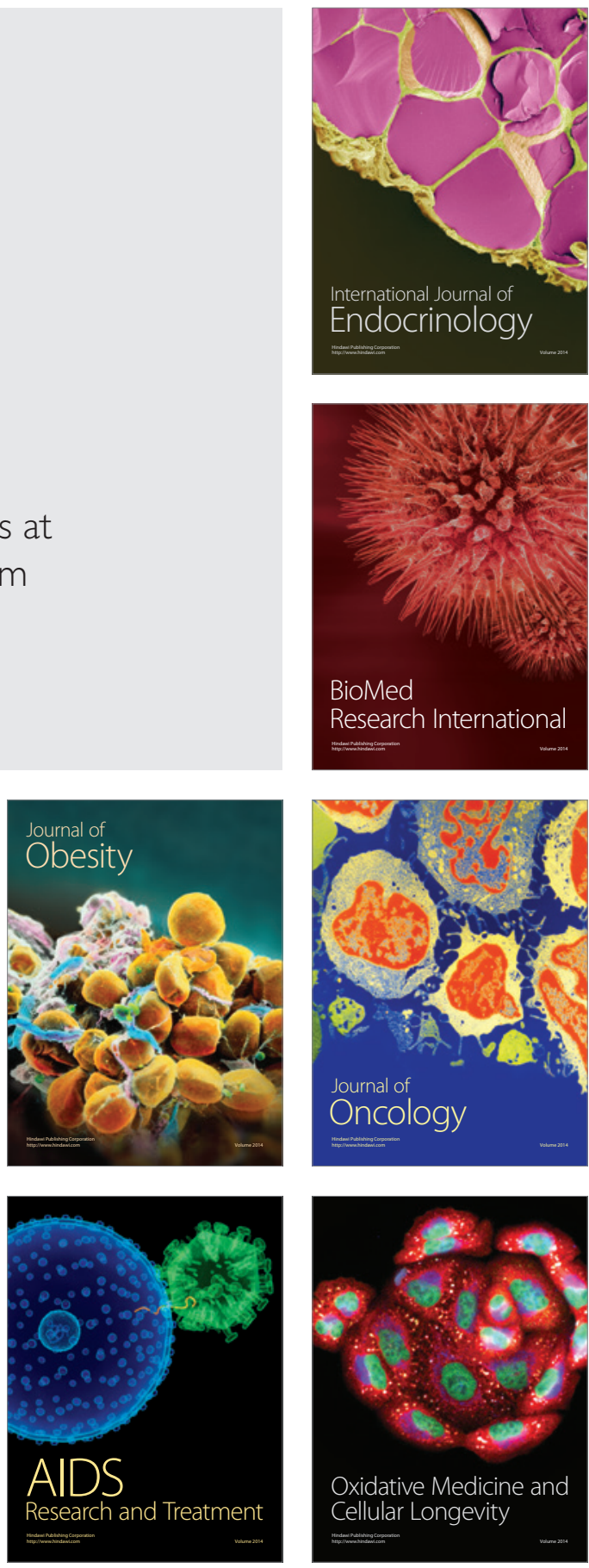